\title{
SMALL SIZE AUVS: OPERATION RESULTS AND NEW MISSION CONCEPTS ${ }^{1}$
}

\author{
Nuno Cruz, Aníbal Matos, João Sousa
}

\author{
Faculdade de Engenharia da Universidade do Porto \\ Rua Dr. Roberto Frias, 4200-465 Porto, Portugal \\ \{nacruz, anibal, jtasso\}@fe.up.pt
}

\begin{abstract}
The number of successful missions with autonomous underwater vehicles (AUVs) has been increasing steadily during the last few years, validating this emerging technology as an efficient tool for underwater sampling. At the same time it has served to envisage their operation in more demanding scenarios. This paper presents results from environment monitoring missions with an AUV and new mission concepts for the operation of one or multiple AUVs. These new concepts are the natural evolution of previous work and also the response to the requirements of some of the identified scenarios. For each type of mission, solutions for navigation, control, coordination, and communications are presented.

Copyright (C) 2004 IFAC
\end{abstract}

Keywords: Autonomous Underwater Vehicles, Mission Design, Environmental Monitoring.

\section{INTRODUCTION}

Traditional techniques for underwater observation are generally expensive and do not offer a comprehensive coverage, especially as the requirements become extremely demanding. On the other hand, recent progresses in underwater technologies have allowed for robotic systems to become highly versatile and, as a consequence, they have been increasingly adopted as efficient and effective tools for underwater observation and intervention. The Underwater Systems and Technology Lab (LSTS), at the University of Porto, pursues the design and implementation of innovative approaches to underwater observation and intervention by the synergistic interaction between advanced topics in control with the latest developments in underwater technology. The laboratory has been involved in the design and implementation of underwater robotic systems for the last 7 years.

\footnotetext{
1 Partially supported by Programa POCTI Medida 2.3
}

This paper presents results obtained in missions executed with a small size AUV as well as advanced mission concepts to be developed and implemented within the scope of the PISCIS project.

The paper is organized as follows. Section 2 briefly describes the key features of the autonomous underwater vehicles currently operated by LSTS or under development. Section 3 introduces the PISCIS project. Section 4 presents some results from operational missions carried out with an AUV from LSTS. Finally, in section 5, there is a discussion on the advanced mission concepts for AUVs that will be addressed under the PISCIS project.

\section{SMALL SIZE AUVS}

AUVs constitute powerful and effective tools for underwater data gathering. These vehicles operate with no physical link with the surface, carrying a 


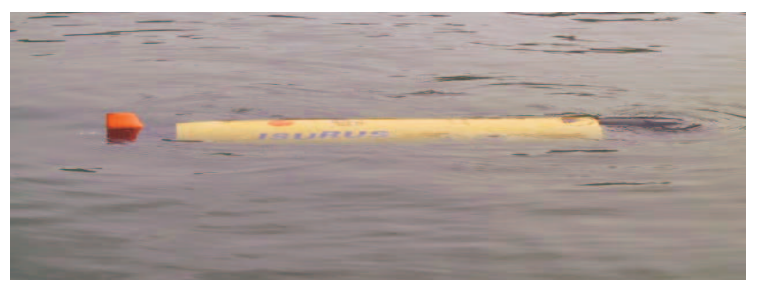

Fig. 1. The Isurus AUV.

set of relevant sensors to characterize the underwater environment.

There is currently a large number of operational AUVs covering scientific, commercial and military applications. The physical characteristics vary greatly according to the expected working environments, payload sensors, propulsion and control solutions, and endurance.

The LSTS has been operating and customizing the Isurus AUV, for the last 6 years (Cruz et al., 1999). Isurus (Fig. 1) is a REMUS class AUV, built by the Woods Hole Oceanographic Institution, MA, USA, in 1997. These vehicles are low cost, lightweight, and specially designed for coastal waters monitoring (von Alt et al., 1994). The reduced weight and dimensions makes them extremely easy to handle, requiring no special equipment for launching and recovery. These features have been of paramount importance to the LSTS strategy of validating most of the developments through an intensive test programme at extremely reduced costs.

Isurus has a torpedo shaped hull, about 1.5 meters long, with a diameter of $20 \mathrm{~cm}$ and weighting about $35 \mathrm{~kg}$ in air. Inside the hull several subsystems have been improved or specifically developed at LSTS, contributing to the enhancement of the vehicle's performance and reliability. The maximum forward speed is 4 knots, being the best energy efficiency achieved at about 2 knots. At this velocity, the energy provided by a set of rechargeable Lithium-Ion batteries may last for over 20 hours (i.e., over 40 nautical miles). Although small in size, this vehicle can accommodate a wide range of oceanographic sensors, such as CTD, Sidescan Sonars, ADCP, or Optical sensors.

The experience obtained from operational missions allowed for an exhaustive characterization of the capabilities and limitations of the complete system. Cooperation with mechanical engineers from INEGI resulted in the identification of a set of key features to allow for significant improvements, resulting in a new design (Fig. 2). The new vehicle is already assembled, is currently being tested, and is expected to be executing operational missions in the summer of 2004. These improvements in the mechanical design include: utilization of lighter composite materials in the central hull, saving valuable weight to incorporate new sensors and electronics; increased modularity to ease the reconfigurability of the vehicle depending on the missions to be performed; and incorporation of a radio link to allow for wireless communication when the vehicle is at the surface, avoiding recovery for data transfer.

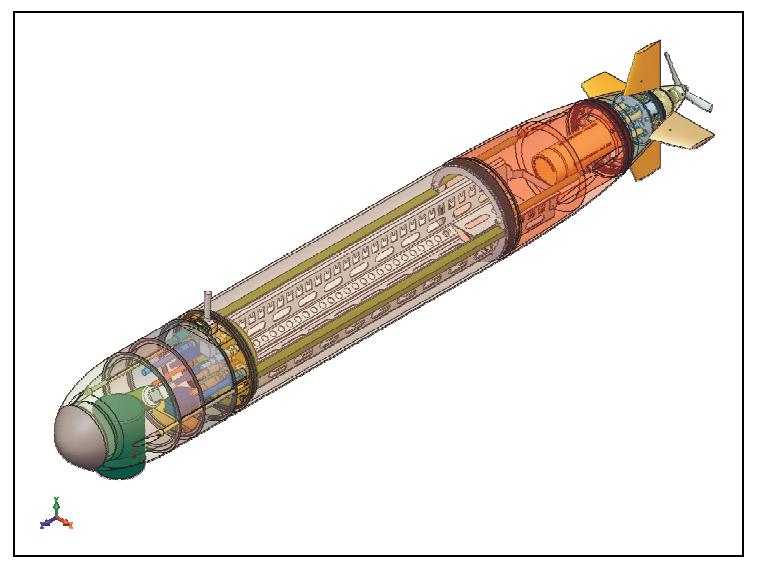

Fig. 2. New generation AUV from LSTS.

\section{THE PISCIS PROJECT}

The PISCIS project started in December 2002, has a total duration of 3 years, and is funded by Programa POCTI Medida 2.3. PISCIS is a joint project involving the Administração dos Portos do Douro e Leixões (APDL), Faculdade de Engenharia da Universidade do Porto, Instituto Superior de Engenharia do Porto and Centro Interdisciplinar de Investigação Marinha e Ambiental (CIIMAR). APDL is the port authority in Porto and is mainly interested in operations in the vicinity of the harbors, involving sidescan sonar missions and other bottom topography studies. Researchers from CIIMAR are particularly interested in oceanographic processes, thus requiring other classes of sensors. Overall, the PISCIS system has to be configurable for applications in real time oceanography, bathymetry, underwater archaeology, and effluents monitoring. Each AUV will integrate a set of relevant sensors for data collection, such as sidescan sonar, CTD, ADCP, fluorometer or other optical sensors. The envisaged accuracy of the navigation system also demands the incorporation of appropriate sensors, such as inertial units and Doppler velocimeters.

The most relevant milestones of this project can be summarized as:

- construction of a new autonomous underwater vehicle;

- simultaneous navigation of several vehicles in the same area of operation;

- coordinated operation of multiple vehicles; and

- specification and control of sensor based missions. 


\section{MISSION RESULTS}

The first operational missions with Isurus took place in 1998, in the estuary of the river Minho, in the northern border between Portugal and Spain. During autonomous missions, typically longer than one hour, the vehicle continuously collected CTD and bathymetric data, while navigating on a long baseline (LBL) transponder network (Matos et al., 1999).

During the missions in the river Minho, the vehicle and the acoustic transponders were deployed and recovered by 2 people from a small fishing boat, which demonstrated the reliability and the operational effectiveness of the system (Cruz et al., 1999). Since then, several other similar missions have been performed on different scenarios. A different problem addressed with the Isurus AUV was the evaluation of the environmental impact of the heated discharge of a power plant located near the Crestuma dam in the river Douro. This included the influence in the mixing process of the river bed, the free surface and the interaction with bottom topography. For this analysis, the AUV was programmed to collect CTD data near the discharge outfall as well as bathymetric data (Ramos et al., 2000) in the neighboring area.

In the summer of 2002, on an innovative mission, the vehicle was employed on a monitoring plan for a sewage outfall, $3 \mathrm{~km}$ off the Portuguese coast, near Aveiro. A simulation model was used in the first place to predict the location of the plume rising from the outfall, given the characteristics of the sewage and the surrounding water (density, currents, etc.). The Isurus AUV was then programmed to collect CTD data at different depths in and out of the plume. Even under severe sea conditions, with wave heights above 4 meters, the vehicle successfully performed the 2 hours mission according to plan (Ramos et al., 2002). Fig. 3 illustrates temperature and salinity data collected by the vehicle, 2 and 4 meters bellow surface.

During the mission, the vehicle motion was monitored using an external tracking system (Cruz et al., 2001). This system computes the position of the vehicle in real time by passively listening to the acoustic signals exchanged between the vehicle and the acoustic navigation beacons.

After the mission, a trajectory smoothing algorithm (Matos et al., 2003) was implemented to improve the accuracy of the navigation system in order to better locate the data collected throughout the mission. Overall, the vehicle position accuracy is about 1 to 3 meters, being limited by the uncertainty is the location of the navigation beacons.
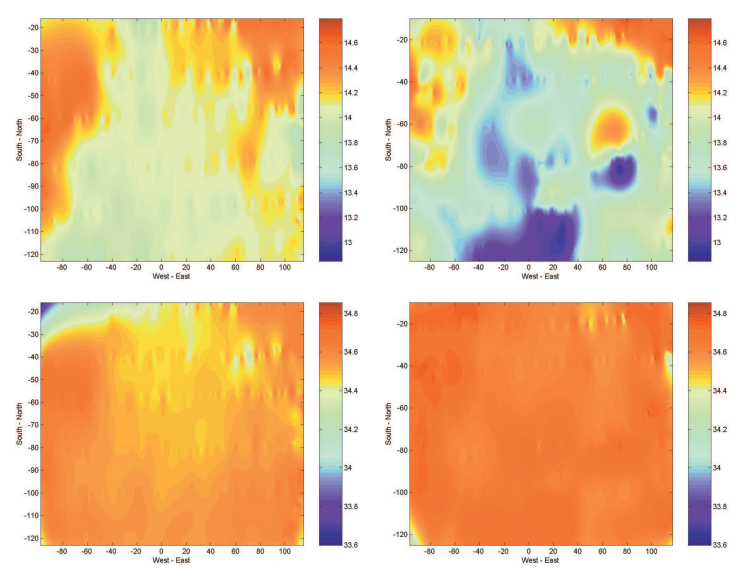

Fig. 3. Temperature and salinity data.

\section{NEW MISSION CONCEPTS}

Most of the current AUV applications consist in the replacement of traditional technology in order to reduce sampling time. There is also an increasing number of successful missions where AUVs are the only technological solution, such as under ice and other hazardous environments. At the same time, these successes have served to envisage the operation of AUVs in more demanding scenarios.

These scenarios impose design, implementation and reliability requirements that can hardly be fulfilled with traditional mission concepts. The following subsections address some of these scenarios and describe the solutions envisaged to overcome their main challenges.

\subsection{Multiple AUVs}

The use of multiple AUVs in the same mission can be advantageous as compared to single AUV operation for very different reasons and in several operational scenarios (Willcox et al., 2001). Among the most important ones are lower mission costs (by reducing operating time and the associated logistic costs), reduced coverage time (required for the study of phenomena with high temporal variability and for the collection of spatially distributed synoptic data) and resolution of spatio-temporal evolution ambiguities of data collected on different places at different times.

In the scope of the PISCIS project, multiple vehicles will be employed for the monitoring of a sewage outfall system. Previous data from the Aveiro outfall showed that the emanating plume changes rapidly as compared to the time scale associated to a single vehicle operation. This is therefore an operational scenario where multiple AUVs can be employed with great benefits.

For this scenario, two different approaches will be tested, using two vehicles simultaneously. In the first, both vehicles will have the same mission 
script and will be deployed in the same location (Fig. 4). The second vehicle will be launched shortly after the first one so that it will be possible to infer the temporal variability of the scalar field at each sampling location.

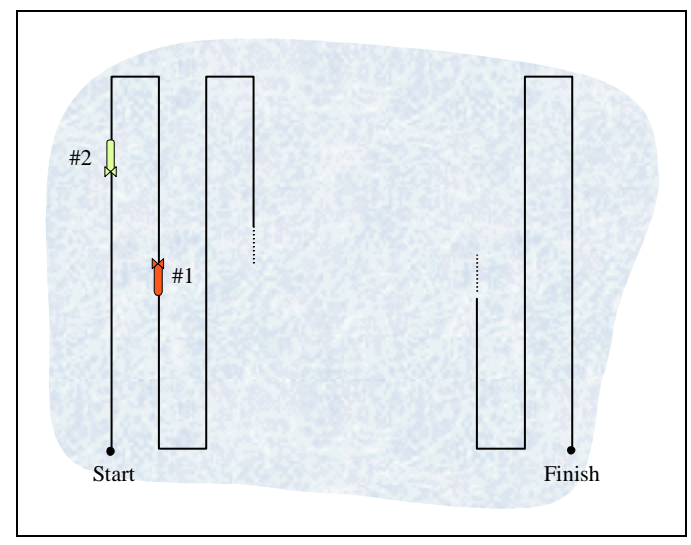

Fig. 4. AUVs with the same path.

In the second approach, each vehicle will cover only half of the desired sampling area (Fig. 5). They will be deployed in different locations and will start their missions at the same time. This way, it will be easier to determine the spatial variation of the scalar field at each instant of time.

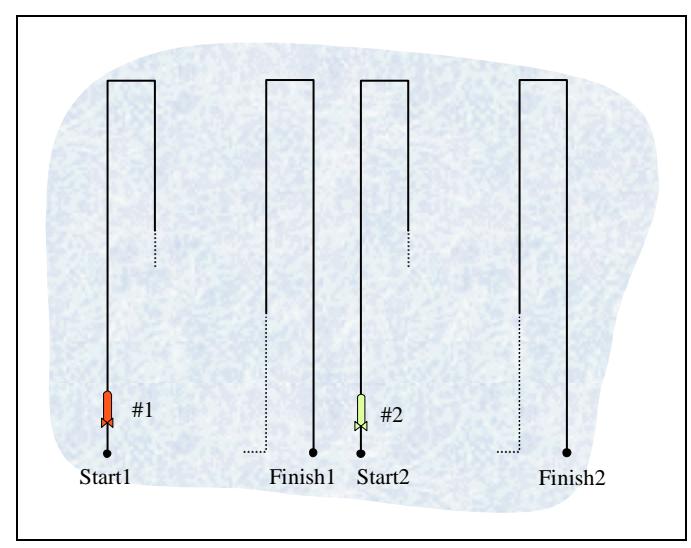

Fig. 5. AUVs in different regions.

In both approaches, there will be no communication link between the vehicles or between each vehicle and a control station, except for very simple acoustic signals similar to the ones used for navigation.

The vehicles will be navigating in long baseline mode using the same acoustic network composed by two acoustic beacons. To determine its position in the horizontal plane, each vehicle determines its distance to each beacon by sending an interrogation signal, waiting for the transponder reply and measuring the time of flight of the acoustic signals. This method is called spherical navigation and does not require additional signal exchanges, or a communication link, for each vehicle to know the position of the other one in real time (Cruz et al., 2001). The versatile beacons previously developed at the LSTS (Cruz et al., 2001) will be deployed for navigation; these beacons can be programmed to simultaneously listen to several interrogation signals and respond to each one with a different signal. To avoid channel conflicts, different signals (single tones) are exchanged between each vehicle and each beacon, totalizing eight different signals. It would be possible for the two vehicles to navigate using a reduced number of acoustic signals, if an hyperbolic navigation algorithm (Bellingham et al., 1992) were used, where the vehicles just listen to signal sent by the beacons. However that solution would require additional signal exchanges for each vehicle to determine the position of the other in real time.

The coordination of the vehicle motions will be achieved by forcing both vehicles to move at the same ground speed. The ground speed is estimated from the navigation data and the control system of each vehicle implements a feedback loop to compute the propeller reference speed based on the deviation between the estimated and desired ground speeds.

To reduce logistics complexity and costs, the mission will be performed with only one operation team, with a small boat. For the first approach, the vehicles will be launched in sequence at the same location, location Start in fig. 4. Each vehicle initiates its mission immediately after having been launched. For the second approach, the operation team initially launches the first vehicle at location Start1, see fig. 5, and then goes to location Start2, where the second vehicle is deployed. In this approach, both vehicles wait for an external acoustic signal to start their missions synchronously. Upon mission completion, each vehicle will circle around the respective finishing point, waiting for recovery.

\subsection{Relocatable Acoustic Network}

Traditionally, the beacons of the acoustic navigation network are moored and remain in the same place during the execution of one or several missions. The number and location of the beacons are selected taking into account the dimensions of the operation area (to ensure enough signal power for detection and avoid adverse geometric configurations), the occlusion of beacons by natural features (to ensure straight line propagation), and the logistics associated to their mooring.

In some scenarios, one or more of these factors greatly affect operational costs, either by requiring a large number of beacons or by increasing the difficulty of beacon deployment. In such cases it is clearly advantageous to use a network of relocatable beacons instead of fixed ones. 


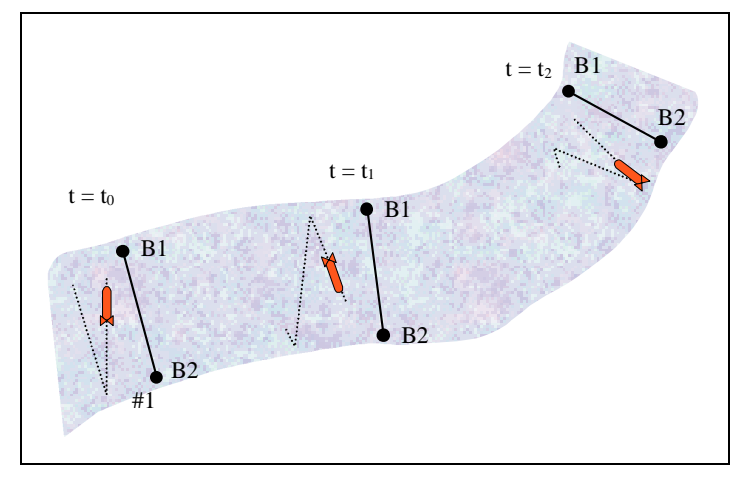

Fig. 6. Relocatable acoustic network.

Within the scope of the PISCIS project, several missions will take place in the rivers Douro and Minho, where one AUV will be collecting CTD and bathymetric data. For a typical mission, the vehicle has to cover an area as wide as the river and a few kilometers long. Both rivers have winding margins, being impossible to cover the operation area with just a few fixed acoustic beacons.

For these missions, navigation will be based on a relocatable acoustic network using two beacons. Each beacon will be attached to a small boat that will move slowly along the river, remaining close to a margin. The motion of the boats will be coordinated such that both of them will continuously define a river cross section.

Since the navigation network is moving, the vehicle does not navigate with respect to an earth fixed frame, but navigates in the frame defined by the two navigation beacons. In this moving frame, the trajectory of the vehicle is just a line, parallel to the baseline and at a given distance from it. During the mission, the vehicle just goes forth and back along this line. Since the baseline is moving, the vehicle will approximately describe a zigzag pattern along the river. Figure 6 shows three pieces of such trajectory, as well as the location of the baseline at three different times.

The vehicle will navigate in LBL mode, measuring its distance to each beacon at a time. To determine its position in the moving frame, the vehicle requires the length of the baseline. Since the width of the river is not constant and the baseline length is related to such width, a simple signal exchanging mechanism between the two beacons will allow for the vehicle to compute in real time the length of the baseline. Although the vehicle does not navigate in a fixed frame, the absolute position of the beacons will be logged throughout the mission so that all measurements can be spatially located upon mission completion.

\subsection{Sensor Based Missions}

Another mission concept being addressed by the PISCIS project is to replace the standard pre- defined AUV mission by an adaptive sampling mission, for which a set of rules is given, but where the trajectories followed by each vehicle are not known a priori. Real time adaptive sampling has been suggested as a way to improve the efficiency in the sampling process, by concentrating the measurements in a region of interest identified according to the local characteristics of the scalar field being measured by the vehicle (Consi et al., 1994; Burian et al., 1996; Willcox et al., 2001). However, only recently the first steps were taken towards implementation of adaptive sampling strategies on AUVs (Barat and Rendas, 2003; Bachmayer and Leonard, 2002; Farrell et al., 2003). Such an approach exploits the onboard computational power to make assumptions about the oceanographic environment and to react to this environment by making decisions about the best sampling strategy to use. By continuously interpreting collected data, this decision can be made in real time so that the vehicle can use most of the available resources (mainly power) in sampling the ocean in regions of interest.

The adaptive sampling paradigm can be implemented in several different ways: first, by adjusting mission parameters according to results from previous runs; second, by feeding the guidance system with new way points as soon as significant data is processed; and finally, by using sensor data directly in the control loop, which requires a good model of the oceanographic phenomena to be effective.

This type of mission raises important issues related to navigation, since the transponder coverage has to include all the region the vehicles are likely to visit during the search/follow process. It is also worth mentioning that the mission management system has to deal with the fact that new "commands" are generated throughout the mission. This may prevent a thorough preliminary validation of the mission plan by simulation.

For the PISCIS project, the "adaptive sampling" concept will be implemented to track and map the thermocline. We will define a region of interest and follow standard $x-y$ navigation in the horizontal plan. As far as depth is concerned, the vehicle will follow a yo-yo pattern, for which the amplitude, offset and frequency are dictated by the scalar temperature measurements (Fig. 7). The main objective is for the vehicle to keep crossing the thermocline, identifying its key parameters: sea surface temperature, mixed layer depth, thermocline depth, thermocline temperature gradient and deep layer stratification. We will follow a parametric model based on a single type profile, similar to the models proposed in (Chu et al., 1997) and (Chu et al., 1999). The first application scenario will be a dam reservoir, where we 


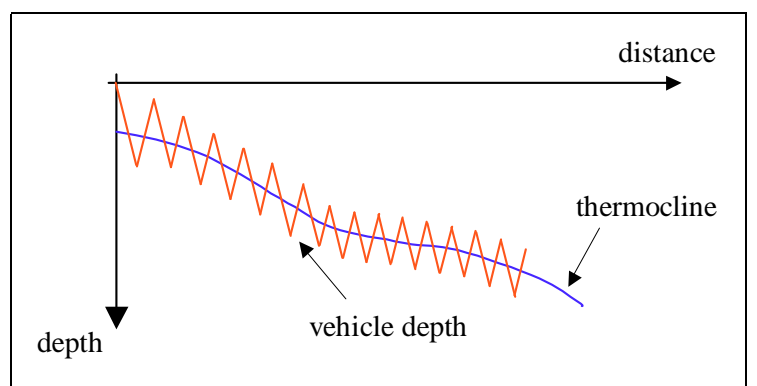

Fig. 7. Thermocline tracking AUV.

will use the Isurus AUV to track and identify the parameters of the seasonal thermocline, during the summer of 2004.

\section{CONCLUSIONS}

Over the last years, the LSTS has accumulated valuable expertise in several areas related to underwater systems. So far, the major technical developments at the laboratory have been driven by the requirements identified in real applications and most of the solutions obtained have actually been validated in successful operational missions. Such experience and know-how is currently being applied in new projects with demanding requirements, such as the case of the PISCIS project, a low cost integrated system for rapid assessment of the underwater environment. Under this project, several mission scenarios were envisaged, for which the main challenges were identified and so were the methods to tackle such challenges, in the shape of new paradigms for system integration and operation.

\section{REFERENCES}

Bachmayer, R. and N. E. Leonard (2002). Vehicle networks for gradient descent in a sampled environment. In: Proceedings of the IEEE Conference on Decision and Control CDC'02. Las Vegas, NV, USA.

Barat, C. and M. J. Rendas (2003). Benthic boundary tracking using a profiler sonar: A mixture model approach. In: Proc. of the MTS/IEEE Conf. Oceans'03. San Diego, CA, USA.

Bellingham, J. G., T. R. Consi, U. Tedrow and D. Di Massa (1992). Hyperbolic acoustic navigation for underwater vehicles: Implementation and demonstration. In: Proc. of the IEEE Symp. on AUV Techn. AUV'92. Washington DC, USA.

Burian, E., D. Yoerger, A. Bradley and H. Singh (1996). Gradient search with autonomous underwater vehicles using scalar measurements. In: Proc. of the IEEE Symp. on AUV Techn. $A U V^{\prime} 96$. Monterey, CA, USA.
Chu, P. C., C. R. Fralick Jr., S. D. Haeger and M. J. Carron (1997). A parametric model for the yellow sea thermal variability. $A G U J$. Geoph. Res. 102(C5), 10499-10507.

Chu, P. C., Q. Wang and R. H. Bourke (1999). A geometric model for the Beaufort/Chukchi sea thermohaline structure. J. Athm. and Ocean. Tech. 16(6), 613-632.

Consi, T. R., J Atema, C. A. Goudey, J. Cho and C. Chryssostomidis (1994). AUV guidance with chemical signals. In: Proc. of the IEEE Symp. on AUV Techn. AUV'94. Cambridge, MA, USA.

Cruz, N., A. Matos, A. Martins, J. Silva, D. Santos, D. Boutov, D. Ferreira and F. Pereira (1999). Estuarine environment studies with Isurus, a REMUS class AUV. In: Proc. of the MTS/IEEE Conf. Oceans'99. Seattle, WA, USA.

Cruz, N., L. Madureira, A. Matos and F. L. Pereira (2001). A versatile acoustic beacon for navigation and remote tracking of multiple underwater vehicles. In: Proc. of the MTS/IEEE Conf. Oceans'01. Honolulu, HI, USA.

Farrell, J., W. Li, S. Pang and R. Arrieta (2003). Chemical plume tracing experimental results with a REMUS AUV. In: Proc. of the MTS/IEEE Conf. Oceans'03. San Diego, $\mathrm{CA}, \mathrm{USA}$.

Matos, A., N. Cruz, A. Martins and F. Pereira (1999). Development and implementation of a low-cost LBL navigation systems for an AUV. In: Proc. of the MTS/IEEE Conf. Oceans'99. Seattle, WA, USA.

Matos, A., N. Cruz and F. L. Pereira (2003). Post mission trajectory smoothing for the Isurus AUV. In: Proc. of the MTS/IEEE Conf. Oceans'03. San Diego, CA, USA.

Ramos, P., M. Neves and F. Pereira (2002). Using near field model predictions to detect and map an outfall sewage plume with an autonomous underwater vehicle. In: Proc. of the MTS/IEEE Conf. Oceans'02. Biloxi, MI, USA.

Ramos, P., M. Neves, N. Cruz and F. L. Pereira (2000). Outfall monitoring using autonomous underwater vehicles. In: Proc. of the Int. Conf. Marine Wastewater Discharges $M W W D$ '2000. Génova, Italy.

von Alt, C., B. Allen, T. Austin and R. Stokey (1994). Remote environmental measuring units. In: Proc. of the IEEE Symp. on AUV Techn. AUV'94. Cambridge, MA, USA.

Willcox, J. S., J. G. Bellingham, Y. Zhang and A. B. Baggeroer (2001). Performance metrics for oceanographic surveys with autonomous underwater vehicles. IEEE J. Ocean. Eng. 26(4), 711-725. 